\title{
p53 spreads out further: suppression of EMT and stemness by activating miR-200c expression
}

\author{
Jörg Schubert ${ }^{1}$, Thomas Brabletz ${ }^{1,2}$ \\ ${ }^{1}$ Department of Visceral Surgery, ${ }^{2}$ Comprehensive Cancer Center, University of Freiburg Medical Center, 79106 Freiburg, Germany \\ Cell Research (2011) 21:705-707. doi:10.1038/cr.2011.62; published online 12 April 2011
}

There is no need to emphasize the overwhelming importance of p53 for crucial cellular processes and of genetic alterations resulting from its loss of function or a dominant oncogenic mutant in many human cancer types. These functions are well described and include control of cell cycle progression, apoptosis, senescence, DNA-repair mechanisms and autophagy [1].

A new page in the huge book of p53 was opened in a recent publication by Chang et al. by linking p 53 to the control of an epithelial-mesenchymal transition (EMT) and its associated effects [2]. EMT is a key program in embryonic development and is conferred by so-called EMT activators, transcriptional repressors, such as Snail and ZEB family members, which suppress expression of epithelial genes. Its aberrant activation in cancer cells induces malignant tumor progression, invasion, dissemination and finally metastasis, due to acquisition of an abnormal cellular motility [3]. Recently, EMT was also linked to an activation of stemness properties, thereby conferring a combined stemness and motility phenotype to cancer cells [4]. Thus aberrant EMT might induce a subpopulation of "Migrating Cancer Stem Cells" as potential source of metastasis [5]. At

Correspondence: Thomas Brabletz

Tel: +49-761 270 2577; Fax: +49-761 2702579

E-mail: thomas.brabletz@uniklinik-freiburg.de molecular level EMT activators were shown to suppress transcription of all five members of the miR-200 family of microRNAs, located in two clusters on Chr. 1 (miR-200a, b and 429) and Chr. 12 (miR-141 and 200c) [6, 7]. In turn, miR-200 members inhibit translation of EMT-activators, particularly ZEB factors, thereby inducing the reverse process of a mesenchymal-epithelial transition (MET) [7-10]. Thus, miR-200 and ZEB1 factors are linked in a reciprocal feed-back loop, which controls an immense phenotypic flexibility and plasticity [11]. The range of this plasticity became clear, when it was shown that miR-200 not only counteracts classical EMT properties like cell motility, but also suppresses translation of stem cells factors, such as BMI1 $[12,13]$. Accordingly, miR-200 family members were shown to be down-regulated in breast cancer stem cells and normal breast epithelial stem cells [12].

Due to the strong potency of miR200 family members, in particular miR200c, to control crucial cellular processes, such as motility and stemness, its own regulators play an important role. Although EMT activators, particularly ZEB factors, were already identified as transcriptional repressors of miR-200 genes, their activating pathways were still undefined. This gap was closed in the publication by Chang et al. [2], showing that $\mathrm{p} 53$ is a transcriptional activator of the miR-200c gene (Figure 1).
Knowing that miR-200 family members are down-regulated in the stem cell fraction of primary human mammary epithelial cells (HMECs), the authors looked for regulatory elements in the respective promoters. They detected p53 response elements in the common promoter of miR-141 and miR-200c (but not in the second cluster encoding miR-200a, b and 429) and validated a direct transcriptional activation by p53. Overexpression of p53 increased expression of miR-200c and reduced expression of its known targets BMI1, KLF4 and ZEB1. Importantly, along with a 53 -dependent activation of miR200c, stemness and EMT properties were suppressed. In contrast, shRNAmediated depletion of $\mathrm{p} 53$ or inhibition of WT p53 expression by TGF $\beta$ treatment in MCF12A breast epithelial cells had the opposite effect. Is miR-200c an important effector of p53 functions? This was verified by showing that the consequences of p53 depletion on EMT and stemness could be rescued by overexpressing miR-200c. In contrast, the suppression of EMT and stemness by overexpressing WT p53 could be reversed by inhibition of endogenous miR-200c function by using specific antagomirs.

As p53 is a crucial tumor suppressor and EMT/stemness is implicated as an important aspect in tumor initiation and malignant progression, the authors further analyzed the impact of miR- 


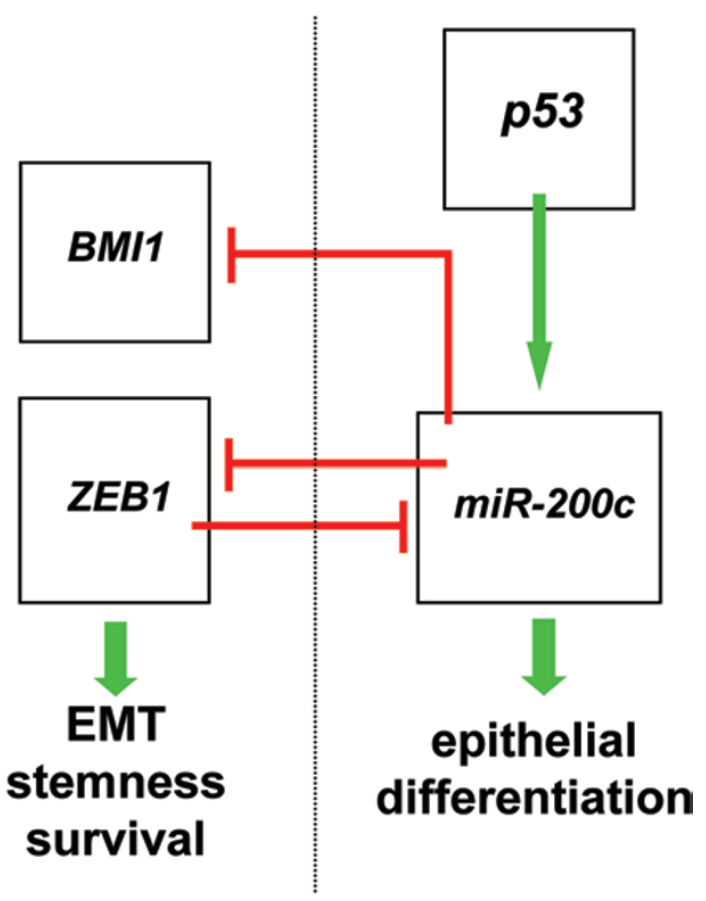

Figure 1 p53 activates transcription of miR-200c. ZEB1 and miR-200 family members repress expression of each other and are linked in a reciprocal feedback loop. In addition miR-200 inhibits translation of stem cell factors, such as BMI1. p53 stimulates expression of miR-200c, thereby driving epithelial differentiation and counteracting EMT and stemness. miR-200c expression can be inhibited in cancer cells by aberrant expression of EMT-factors, such as ZEB1 or p53 mutations, triggering an EMT and stemness phenotype.

200c activation by p53 in cancer cells. Overexpression of four different oncogenic mutants of p53 (R175H, R249S, $\mathrm{R} 273 \mathrm{H}, \mathrm{R} 280 \mathrm{~K}$ ) in MCF12A, expressing only WT p53, induced a down-regulation of miR-200c and a subsequent up-regulation of ZEB1, BMI1 as well as an increase in the $\mathrm{CD} 24^{-} \mathrm{CD} 44^{+}$stem cell fraction. Importantly, overexpression of WT p53 in BT549 breast cancer cells (harbouring the $\mathrm{p} 53^{\mathrm{R} 249 \mathrm{~S}}$ mutant) could compete with the oncogenic mutant, resulting in increased miR-200c expression and a reduced CD24- $\mathrm{CD} 44^{+}$ stem cell fraction. Finally the clinical relevance was validated in 106 cases of human breast cancers by demonstrating a correlation of mutant $\mathrm{p} 53$ expression with high tumor grading, high levels of ZEB1 and BMI1, as well as reduced levels of miR-200c expression.
The study of Chang et al. is exciting because the impact of this discovery for cancer biology is far beyond a p53-dependent regulation of miR-200 genes. It links the most important genetic alteration in human cancer to the emerging fields of EMT and cancer stem cells and their importance for metastasis. Thereby it fosters the role of p53 in controlling stemness properties. Recently, Cicalese et al. demonstrated a link between loss of p53 function and increased symmetric divisions of potential cancer stem cells in a transgenic mouse model of breast cancer [14]. Moreover it was already shown that microRNAs are involved in the control of other p53-dependant functions. Several groups demonstrated that cell cycle arrest, apoptosis and senescence can be triggered by $\mathrm{p} 53$-induced transcriptional activation of the microRNAs, miR-34a and $\mathrm{miR}-34 \mathrm{~b} / \mathrm{c}$ [15]. Besides its obvious role in tumor progression the results also indicate a physiological role of the p53/miR-200c link. Is p53-mediated activation of miR-200c essential for the control of stemness vs. differentiation during embryonic development of epithelial tissues and for their adult homeostasis? Further work should address these issues.

One result of the publication, showing that both overexpression of dominant active mutant p53, as well as depletion of normal WT $\mathrm{p} 53$, leading to its complete loss of function, can inhibit miR-200c expression and its associated functions, raises additional questions. This stands in contrast to many publications describing specific oncogenic effects of mutant p53 in human cancers, which can not be achieved by a complete 553 loss. For instance, only mutant p53, not a complete loss, promotes invasion and metastasis in a genetic mouse model of pancreatic cancer, whereas the complete loss of p53 also induces an escape of senescence and growth arrest [16]. This indicates that other, additional mechanisms of dominant $\mathrm{p} 53$ mutations in driving EMT, tumor invasion and metastasis must exist. Again the control of microRNA function might be involved: WT $\mathrm{p} 53$ activates only one of the two miR-200 clusters. How is the second cluster activated, and more importantly how it is inactivated in tumors with mutated p53? Is it only due to aberrant activation of EMT inducers, such as ZEB1? Or are additional effects of mutant p53 involved? Cancers often show a general decline in microRNA maturation and expression, which might be due to a functional suppression of microRNA maturation factors. Recently p53 was shown to enhance microRNA maturation by associating with Drosha [17]. Has mutant p53 a dominant function in inhibiting microRNA maturation, which cannot be exerted by a simple complete loss of p53 function? Further work should address, if this is another 
oncogenic aspect of mutant p53.

\section{References}

1 Vousden KH, Prives C. Blinded by the light: the growing complexity of p53. Cell 2009; 137:413-431.

2 Chang CJ, Chao CH, Xia W, et al. p53 regulates epithelial-mesenchymal transition and stem cell properties through modulating miRNAs. Nat Cell Biol 2011; 13:317-323.

3 Thiery JP, Acloque H, Huang RY, Nieto MA. Epithelial-mesenchymal transitions in development and disease. Cell 2009; 139:871-890.

4 Mani SA, Guo W, Liao MJ, et al. The epithelial-mesenchymal transition generates cells with properties of stem cells. Cell 2008; 133:704-715.

5 Brabletz T, Jung A, Spaderna S, Hlubek F, Kirchner T. Opinion: migrating cancer stem cells - an integrated concept of malignant tumour progression. Nat Rev Cancer 2005; 5:744-749.

6 Bracken CP, Gregory PA, Kolesnikoff $\mathrm{N}$, et al. A double-negative feedback loop between ZEB1-SIP1 and the mi-
croRNA-200 family regulates epithelial-mesenchymal transition. Cancer Res 2008; 68:7846-7854.

7 Burk U, Schubert J, Wellner U, et al. A reciprocal repression between ZEB1 and members of the miR-200 family promotes EMT and invasion in cancer cells. EMBO Rep 2008; 9:582-589.

8 Gregory PA, Bert AG, Paterson EL, et al. The miR-200 family and miR-205 regulate epithelial to mesenchymal transition by targeting ZEB1 and SIP1. Nat Cell Biol 2008; 10:593-601.

9 Korpal M, Lee ES, Hu G, Kang Y. The miR-200 family inhibits epithelialmesenchymal transition and cancer cell migration by direct targeting of E-cadherin transcriptional repressors ZEB1 and ZEB2. J Biol Chem 2008; 283:14910-14914.

10 Park SM, Gaur AB, Lengyel E, Peter ME. The miR-200 family determines the epithelial phenotype of cancer cells by targeting the E-cadherin repressors ZEB1 and ZEB2. Genes Dev 2008; 22:894-907.

11 Brabletz S, Brabletz T. The ZEB/miR200 feedback loop--a motor of cellular plasticity in development and cancer? EMBO Rep 2010; 11:670-677.

12 Shimono Y, Zabala M, Cho RW, et al. Downregulation of miRNA-200c links breast cancer stem cells with normal stem cells. Cell 2009; 138:592-603.

13 Wellner U, Schubert J, Burk UC, et al. The EMT-activator ZEB1 promotes tumorigenicity by repressing stemnessinhibiting microRNAs. Nat Cell Biol 2009; 11:1487-1495.

14 Cicalese A, Bonizzi G, Pasi CE, et al. The tumor suppressor p53 regulates polarity of self-renewing divisions in mammary stem cells. Cell 2009; 138:1083-1095.

15 Hermeking H. p53 enters the microRNA world. Cancer Cell 2007; 12:414418.

16 Morton JP, Timpson P, Karim SA, et al. Mutant p53 drives metastasis and overcomes growth arrest/senescence in pancreatic cancer. Proc Natl Acad Sci USA 2010; 107:246-251.

17 Suzuki HI, Yamagata K, Sugimoto $\mathrm{K}$, Iwamoto $\mathrm{T}$, Kato S, Miyazono K. Modulation of microRNA processing by p53. Nature 2009; 460:529-533. 